\title{
A Method for Evaluating Soluble Redox Couple Stability using Microelectrode Voltammetry
}

\author{
Jeffrey A. Kowalski ${ }^{1,2}$, Alexis M. Fenton Jr. ${ }^{2}$, Bertrand J. Neyhouse ${ }^{1,2}$, and Fikile R. Brushett ${ }^{1,2, z}$ \\ ${ }^{1}$ Joint Center for Energy Storage Research, Massachusetts Institute of Technology, Cambridge, MA 02139 \\ ${ }^{2}$ Department of Chemical Engineering, Massachusetts Institute of Technology, Cambridge, MA 02139 \\ ${ }^{2}$ Corresponding author: brushett@mit.edu
}

\begin{abstract}
Soluble, redox-active, organic materials hold promise as charge-storage species for flow batteries; however, their stability during extended operation remains a key challenge. While a number of spectroscopic and electrochemical techniques are currently used to probe these complex and often ill-defined decay pathways, each technique has limitations, including accessibility and direct evaluation of practical electrolytes without preparatory steps. Here, we use microelectrode voltammetry to directly observe nonaqueous flow battery electrolytes, simultaneously identifying the rate of charged materials decay (reversible material loss) and total material decay (irreversible material loss). We validate this technique using ferrocene as a stable model redox couple, examine and address sources of error, and finally, demonstrate its capability by assessing the decay of a well-studied and moderately-stable substituted dialkoxybenzene [2,5-di-tert-butyl-1,4-bis(2methoxyethoxy)benzene]. These results suggest that microelectrodes may have utility for rapid assessment of redox electrolyte state-of-charge and state-of-health, both in-operando and postmortem.
\end{abstract}

Keywords: microelectrodes, voltammetry, organic redox couples, energy storage 


\section{Introduction}

Redox flow batteries (RFBs) are a promising electrochemical technology for energy-intensive grid storage, ${ }^{1}$ but further cost reductions are needed for widespread adoption, spurring research efforts into new charge storage chemistries and reactor designs. ${ }^{2-5}$ Notably, the recent emergence of redox active organic molecules offers intriguing new pathways to low-cost energy storage through tunable molecular structure and inexpensive synthesis routes. ${ }^{6-8}$ While the rapid and continued advancement of these redox couples is exciting, many of these compounds are prone to decompose in electrolyte. ${ }^{9-11}$ Iterative design-test-improve cycles have successfully addressed these stability issues, but this procedure is difficult to employ as molecules become increasingly robust and failure modes become more subtle. ${ }^{6,12}$ Thus, there is a growing need for more advanced diagnostic techniques to evaluate materials performance, particularly stability.

At present, the most common method for assessing redox active species stabilities in RFBs is extensive charge/discharge cycling in an electrochemical cell to correlate capacity fade to overall species decay. ${ }^{9,13-20}$ However, in addition to being time-consuming, there are no standardized cycling protocols, which challenges meaningful comparisons of stability data across the literature. ${ }^{21}$ An alternative approach, which is sometimes coupled with cell cycling, is interrogation of a particular reporter constituent by spectroscopy techniques to not only monitor decay rates but also elucidate chemical information relevant to mechanisms of decomposition. Examples of such techniques include electron paramagnetic resonance (EPR) to track the decomposition kinetics of radical species, ${ }^{22-24}$ nuclear magnetic resonance (NMR) to identify decay products and monitor decomposition kinetics, ${ }^{6,10,19,25}$ and UV-Vis spectroscopy to probe the chemical stability of charged species. ${ }^{14,26}$ While potentially powerful, these analytical methods are often limited by their compatibility with complex multicomponent electrolyte solutions and, consequently, specialty 
chemicals (e.g., radiolabeling) and preparatory steps (e.g., dilutions, separations, etc.) are used to facilitate data analysis. These requirements not only add experimental cost, complexity, and time, but also impact the processes that can be reliably assessed that may vary depending on cell chemistry. Further, typical electroanalytical techniques (e.g., cyclic voltammetry) can be leveraged to more easily obtain in operando data, but the signals from many of these techniques are convoluted by $i R$ losses at high concentrations.

Microelectrode voltammetry may address some of the limitations described above, providing information on species decay rates in complex electrochemical environments across a wide range of component concentrations. ${ }^{27}$ The voltammetric response of electrodes in quiescent electrolytes is based on the interplay between the rates of diffusive transport to and from the surface and the reaction kinetics on that surface, where at large enough overpotentials, the reactions limited by the rate of diffusion. The reactant transport for disk macroelectrodes (radius $\sim 1 \mathrm{~mm}$ ) is well described by semi-infinite linear diffusion, as the electrode radius is significantly larger than the boundary layer thickness. However, for disk microelectrodes, the smaller electrode radius $(\sim 5 \mu \mathrm{m})$ means that reactant transport is enabled by both linear and radial diffusion. Here, the boundary layer thickness quickly exceeds the electrode radius and transport is dominated by radial diffusion, resulting in constant reactant flux to the electrode surface and thus a steady-state current. Importantly, this steady state behavior leads to a plateau at large overpotentials as the current becomes diffusion-limited. ${ }^{28}$

In addition to this nature of diffusive transport, the scale of microelectrodes leads to several distinct advantages as compared to macroelectrodes. ${ }^{29}$ First, owing to their small surface area, microelectrodes have comparatively low currents and thus $i R$ distortions, which has enabled voltammetry in previously inaccessible media (e.g., high-resistance solutions, electrolytes with 
proportionately low supporting salt concentrations). ${ }^{29}$ Second, charging currents have a minimal effect on microelectrode voltammograms because the capacitance time constant is directly proportional to the electrode radius, thereby increasing the signal-to-noise ratio of microelectrode voltammograms ${ }^{27}$ Additionally, the steady state behavior exhibited in the current response allows the oxidative and reductive currents to be measured separately using voltammetry, simplifying the analysis of the electrolyte of interest.

Microelectrodes have primarily been used in electroanalytical chemistry to extract electrochemical properties (e.g. diffusion coefficients, kinetic rate constants), ${ }^{30,31}$ to sense a low concentration electroactive species in various analytes, ${ }^{32-35}$ and to probe complex biological processes. ${ }^{36,37}$ However, to date, their application in energy storage devices has been limited, although it is worth noting that recently Stolze et al. demonstrated the utility of microelectrodes for real-time state-of-charge diagnostics in a symmetric aqueous organic RFB. ${ }^{38}$ We postulate that microelectrodes can be used to assess the chemical stability of a redox couple, thereby enabling an accessible, inexpensive method to determine and compare the decay rates of electroactive materials in electrolyte solutions that approximate those used in practical embodiments.

Here, we present an electrochemical technique that uses microelectrode voltammetry to simultaneously measure charged and total species decay. We start with a brief overview of microelectrode theory and its application to stability studies. Then, using the ferrocene/ferrocenium redox couples as a model system, we assess the relative magnitude and impact of different sources of error, including the measurement changing the bulk concentration, unequal diffusion coefficients, electrode stability, and temperature variations from charging the solution. Finally, we apply this protocol is to a model decay compound, 2,5-di-tert-butyl-1,4-bis(2- 
methoxyethoxy)benzene (DBBB), ${ }^{11,18,30,39}$ to evaluate decay rates across a range of concentration, finding general agreement with prior literature.

\section{Experimental}

An argon-filled glovebox (MBraun Labmaster, $\mathrm{H}_{2} \mathrm{O}<5$ ppm, $\mathrm{O}_{2}<1 \mathrm{ppm}$ ) with an ambient temperature of $26{ }^{\circ} \mathrm{C}$ was used for chemical storage, solution preparation, and experimentation. All materials were used as-received. Solutions were prepared a day in advance of use to ensure complete dissolution of solutes. All electrochemical experiments were conducted in an electrolyte solution consisting of $1 \mathrm{M}$ lithium bis(trifluoromethane)sulfonamide salt (LiTFSI, BASF, > 99.9\%) in propylene carbonate (PC, BASF, > 99.9\%). This composition was selected because of its wide window of electrochemical stability and low volatility. Ferrocene (Sigma Aldrich, 98 \%), ferrocenium hexafluorophosphate (Sigma Aldrich, 97\%), and 2,5-di-tert-butyl-1,4-bis(2methoxyethoxy)benzene (DBBB) were used as redox species. DBBB was synthesized and characterized as previously described., $9,18,39$

To electrochemically generate oxidized species (i.e., ferrocenium, $\mathrm{DBBB}^{+}$), redox electrolyte solutions (solvent, supporting salt, and active species) were charged in a commercial bulk electrolysis cell (BASi, MF-1056) using a VMP-3 potentiostat (Bio-Logic). For these experiments, the solution volume was $30 \mathrm{~mL}$ and the stir rate was $1600 \mathrm{rpm}$. The working, counter, and reference electrodes were reticulated vitreous carbon (BASi), lithium foil (Alfa Aesar, 99.9\%), and fritted lithium foil with $1 \mathrm{M}$ LiTFSI in PC as the fill solution, respectively. A potentiostatic method was used to charge the solution with the potential set at $300 \mathrm{mV}$ above the measured equilibrium potential of the specific redox couple. A coulombic cutoff at the theoretical 100\% state-of-charge was used, and the resulting electrolyte was assumed to be fully charged. For the low concentrations 
tested (1, 3, and $5 \mathrm{mM}$ ), this process took about an hour, whereas charging the $25 \mathrm{mM}$ solution took $c a .3 \mathrm{~h}$. Once the coulombic cutoff was reached, the solution was immediately transferred into two $20 \mathrm{~mL}$ scintillation vials (VWR), $15 \mathrm{~mL}$ per vial, to minimize additional contamination from the counter electrode chamber generated during charging. The solution temperature was measured directly with a glass thermometer (McMaster Carr, $\pm 1^{\circ} \mathrm{C}$ ) after electrolysis and again after all voltammetric measurements were completed. The electrolyte viscosity was independently measured at the determined temperatures using a Viscolite 700 laboratory viscometer (Vindum Engineering).

After the charging step, either square wave voltammetry (SWV) (working electrode: $3 \mathrm{~mm}$ diameter glassy carbon electrode [CH Instruments, Inc.]) or cyclic voltammetry (working electrode: $10 \mu \mathrm{m}$ diameter platinum disc microelectrode [CH Instruments, Inc.]) measurements were taken for $14 \mathrm{~h}$. The SWV measurements were collected every hour on a VMP-3 potentiostat (Bio-Logic) to avoid significantly altering the bulk solution properties, while the microelectrode measurements were collected every 15 min with a CHI-630E (CH Instruments, Inc.). For both types of data collection, the counter and reference electrodes were a platinum coil (BASi, MW1033, 99.95\%), and a fritted $\mathrm{Ag} / \mathrm{Ag}^{+}$cryptand reference electrode with a fill solution of $0.1 \mathrm{M} \mathrm{AgBF}_{4}$ (Sigma-Aldrich, 99.99\%), $0.04 \mathrm{M}$ kryptofix (Acros Organics, 98\%), and $1 \mathrm{M}$ LiTFSI in PC, ${ }^{40}$ respectively. The voltammograms for ferrocene were taken between -0.7 and $-0.2 \mathrm{~V}$ vs. $\mathrm{Ag} / \mathrm{Ag}^{+}$cryptand, while those of DBBB were taken between 0 to $0.5 \mathrm{~V} \mathrm{vs.} \mathrm{Ag} / \mathrm{Ag}^{+}$cryptand. Square wave voltammograms were obtained with a step height of $2 \mathrm{mV}$, a pulse amplitude of $50 \mathrm{mV}$, and a pulse duration of $50 \mathrm{~ms}$, whereas microelectrode cyclic voltammograms were obtained at a scan rate of $10 \mathrm{mV} / \mathrm{s}$. 
Before the first voltammetric measurement of each data set, the electrodes were polished on a MicroCloth pad with $0.05 \mu \mathrm{m}$ alumina powder (Buehler Ltd.). The electrodes were then rinsed with deionized water (Millipore) and wiped dry with lens paper. Because the working, counter, and reference electrodes remained in solution for the duration of the voltammetry experiments, the working electrodes were not polished between each measurement to avoid altering the surface area or position relative to the other electrodes. However, to ensure that any observed decay was not the result of undesirable surface processes occurring on the electrode itself, an additional measurement was taken with a freshly polished electrode after all measurements and compared to the last scan. All experiments were performed in triplicate.

\section{Results and Discussion}

Microelectrode Decay Theory

As previously described, for a microelectrode, diffusion-limited current plateaus are observed at high overpotentials, either much greater or much less than the equilibrium potential. For a microdisc geometry, which experiences radial and axial diffusion, the steady state diffusionlimited current can be described as:

$$
i_{s s}=4 n F c r D
$$

where, $i_{s s}$ is the steady state/plateau current at large overpotentials (A), $n$ is the number of electrons transferred (here, $n=1), F$ is the Faraday constant (96485 C/mol e-), $c$ is the concentration $\left(\mathrm{mol} / \mathrm{cm}^{3}\right), r$ is the microelectrode radius $(0.0005 \mathrm{~cm})$, and $D$ is the diffusion coefficient

$\left(\mathrm{cm}^{2} / \mathrm{s}\right){ }^{28,31}$ In this work, the steady state current was determined by taking the average of the current over a $0.050 \mathrm{~V}$ window, starting $0.075 \mathrm{~V}$ before the voltage limits (for example, if the turnaround potential were $0 \mathrm{~V}$, the steady-state current from $0.075 \mathrm{~V}$ to $0.025 \mathrm{~V}$ would be averaged 
if the potential sweep were negative). We note that this expression is based on dilute solution theory and, in concentrated solutions, non-ideal effects may need to be considered (e.g., migration, diffusional interactions, ion-ion interactions). ${ }^{28,41}$ The magnitudes of the positive and negative steady state currents are directly proportional to the concentrations of the neutral and charged species and, consequently, the total species concentration. This is shown in Figure 1 where the two voltammograms at an initial and arbitrary later time (time $=\mathrm{t}$ ) highlight changes in the steady state currents which can be correlated to changes in species concentrations.

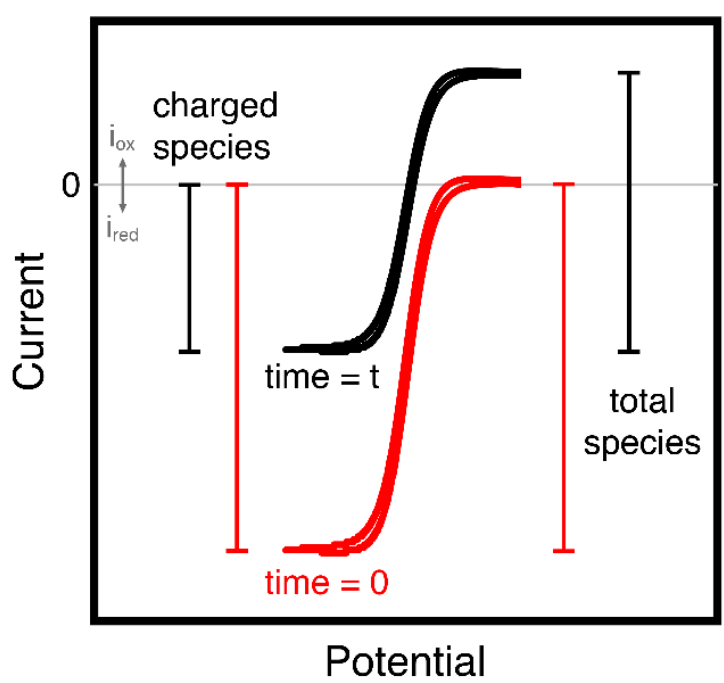

Figure 1: Voltammetry data from the microelectrode experiments at time 0 (red) and some later time, t, (black) showing how to obtain the relative charged species and total species concentrations for an initially oxidized species.

The time-dependence of the microelectrode voltammetry response provides insight into the rate of charged species decay and the manner of this decomposition (either irreversible chemical degradation or reversible discharge). The analysis is based on the initial voltammetry cycle at time $=0$, which serves as a baseline for correlating steady state current measurements to individual and total species concentrations (Figure 1). As the voltammetry profile evolves over time, the instantaneous steady state current measurements can be normalized against this initial response to 
extract the relative concentrations of different species. Specifically, changes in the oxidative and reductive steady state currents can be correlated to concentrations of neutral (reduced) and oxidized species. Further, if the diffusion coefficient of the oxidized and reduced species are assumed to be equal, ${ }^{31}$ the summation of the currents represents the total species concentration. As such, repeated microelectrode voltammetry sweeps of the electrolyte solution enable tracking of both species in the redox couple with a single measurement.

\section{Possible Sources of Error in Decay Analysis using Microelectrodes}

Effective application of this technique requires a systematic assessment of sources of error that may distort analysis. To this end, we identify and evaluate four potential sources of uncertainty: (1) changes in the bulk concentration resulting from the measurements themselves, (2) unequal diffusion coefficients between the oxidized and reduced species, (3) electrode stability, and (4) significant temperature changes during the experiment.

\section{Conversion per Cycle}

As an electrochemical method reliant on mass-transport limited current, each measurement consumes a certain amount of active species that, in turn, can alter the bulk concentration, skewing subsequent measurements and convoluting analysis. Microelectrode voltammetry induces currents on the order of nanoamperes, and as such is not expected to significantly affect the bulk concentration of active species. To confirm this, the number of coulombs converted per cycle must be compared to the total number of coulombs accessible in the solution. As an upper bound for the error due to conversion, we assume that the active species is present in a single oxidation state and 
current at the counter electrode may be ignored. The total number of accessible coulombs in the solution is given by:

$$
Q_{\text {sol }}=n F c V
$$

where $Q_{\text {sol }}$ is the total accessible coulombs in solution (C) and $V$ is the solution volume $\left(\mathrm{cm}^{3}\right)$. For simplicity, we estimate an upper bound for the charge passed per cycle $\left(Q_{c y c}\right)$ as the limiting current multiplied by the time required to complete one cycle $\left(t_{c y c}\right)$ :

$$
Q_{c y c}=i_{s s} t_{c y c}
$$

Taking the ratio of Equation 3 to Equation 2, and combining Equation 1 with Equation 3, yields the maximum fraction of material accessed, $F_{a c c}$, per measurement, shown in Equation 4.

$$
F_{a c c}=\frac{Q_{c y c}}{Q_{s o l}}=\frac{4 r D t_{c y c}}{V}
$$

The upper limit of the fractional conversion can be estimated for studies involving a microelectrode in a solvent of low viscosity operated at a slow scan rate. In this case, the values of $r\left(1 \times 10^{-3} \mathrm{~cm}\right), D\left(1 \times 10^{-4} \mathrm{~cm}^{2} / \mathrm{s}\right), t_{c y c}(100 \mathrm{~s})$, and $V\left(15 \mathrm{~cm}^{3}\right)$ yields an estimated fractional conversion per cycle of approximately $3 \times 10^{-6}$. For context, at this upper bound conversion rate, it would take 3333 cycles before $1 \%$ of the total bulk concentration were altered by the measurements. This corresponds to one voltammogram every 15 minutes for 34 days. Thus, as the experiment duration is only $14 \mathrm{~h}$, any changes in the active species concentrations are not due to the measurement itself.

\section{Unequal Diffusion Coefficients}

In many cases, the diffusion coefficients of the reduced and oxidized species of a redox couple are dissimilar. These, in turn, will result in different magnitudes of steady state current for the 
reduced and oxidized species. However, because independent measurement of both diffusion coefficients may not always be feasible, they are often assumed to be the same, which may lead to an incorrect assessment of species concentrations and impact the total species concentration calculated. ${ }^{31}$ To determine the validity of this assumption, we calculate the error for equal diffusion coefficients as compared to unequal diffusion coefficients for the oxidized and reduced species. First, $R_{c}$, the ratio of the total concentration at any time and the total, initial concentration can be described as:

$$
R_{c}=\frac{c_{r}+c_{o}}{C_{r, i}+C_{o, i}}
$$

where $c_{r}$ is the concentration of the reduced species, $c_{o}$ is the concentration of the oxidized species, $C_{r, i}$ is the initial concentration of the reduced species, and $C_{o, i}$ is the initial concentration of the oxidized species. Substituting Equation 1 into Equation 5 and simplifying yields:

$$
R_{c}=\frac{\left|i_{s s, r}\right| \gamma+\left|i_{s s, o}\right|}{\left|I_{s s, r}\right| \gamma+\left|I_{s s, o}\right|}
$$

where, $i_{s s, r}$ is the reducing steady state current (caused by the oxidized species) at any time, $i_{s s, o}$ is the oxidizing steady state current (caused by the reduced species) at any time, $I_{s s, r}$ is the initial reducing steady state current (caused by the oxidized species), $I_{s s, o}$ is the initial oxidizing steady state current (caused by the reduced species), and $\gamma$ is the ratio of the diffusion coefficient of the reduced species to the diffusion coefficient of the oxidized species $\left(D_{r} / D_{o}\right)$. When the diffusion coefficients are equal, $\gamma$ is unity. If the diffusion coefficients are unequal, the absolute error, $\varepsilon$, can be described as:

$$
\varepsilon=\frac{R_{C, \text { app }}-R_{C, \text { true }}}{R_{C, \text { true }}}=\frac{\frac{\left|i_{s s, r}\right|+\left|i_{s s, o}\right|}{\left|I_{s s, r}\right|+\left|I_{s s, o}\right|}-\frac{\left|i_{s s, r}\right| \gamma+\left|i_{s s, o}\right|}{\left|I_{s s, r}\right| \gamma+\left|I_{s s, o}\right|}}{\frac{\left|i_{s s, r}\right| \gamma+\left|i_{s s, o}\right|}{\left|I_{s s, r}\right| \gamma+\left|I_{s s, o}\right|}}
$$


where $R_{C, a p p}$ is the apparent ratio of total concentration to initial concentration (which inherently assumes $\gamma=1$ ) and $R_{C, \text { true }}$ is the true ratio of total concentration to initial concentration. Simplifying Equation 7 and substituting in $\alpha$ for the ratio of $\left|i_{s s, r}\right|$ to $\left|i_{s, o}\right|$ and $\beta$ for $\left|I_{s s, r}\right|$ to $\left|I_{s s, o}\right|$ yields:

$$
\varepsilon=\frac{(\alpha-\beta)(1-\gamma)}{(\alpha \gamma+1)(\beta+1)}
$$

Note, that from Equation 8, if $\gamma$ is one, the error is zero. Figure 2 plots the error for different $\gamma$ values for a reasonable range of values for $\alpha$ and $\beta$ as determined by experiment. 

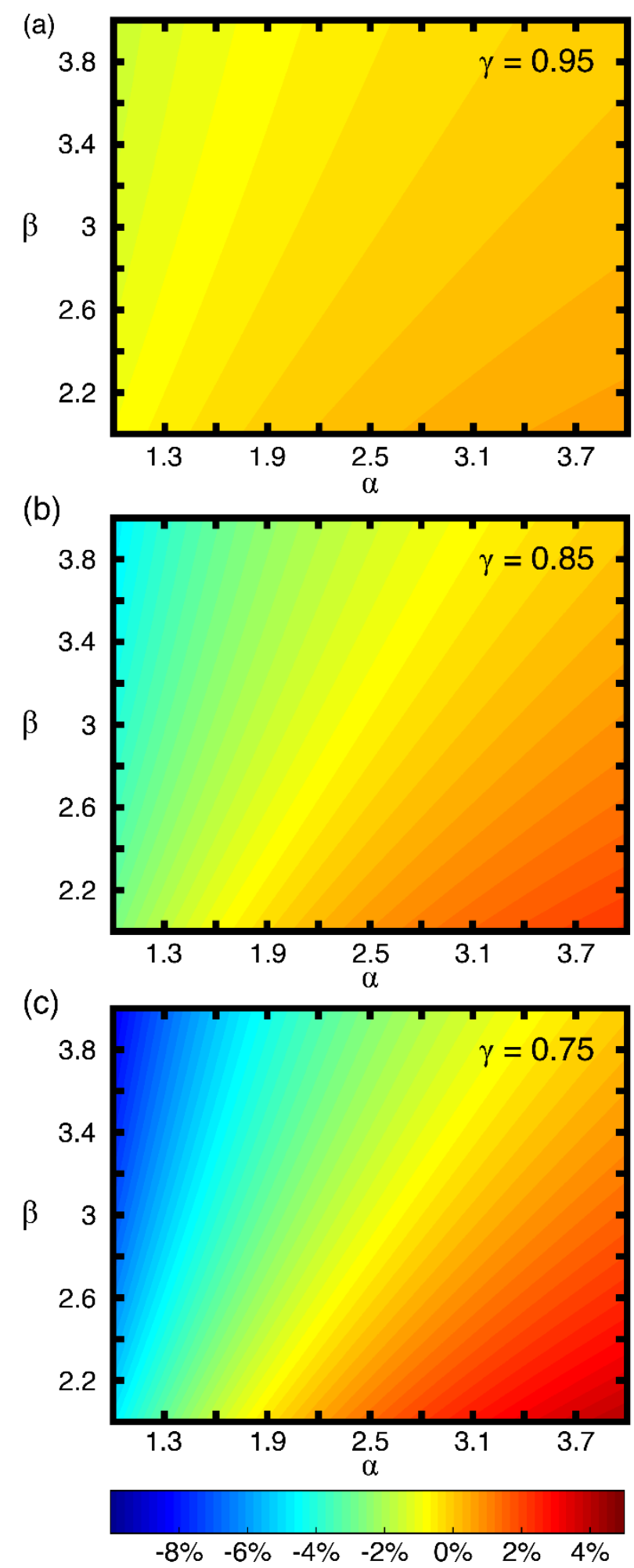

Figure 2: Plots of the error analysis for the total species concentration for $\gamma\left(D_{r} / D_{o}\right)=0.95$ (a), $\gamma=$ 0.85 (b), and $\gamma=0.75$ (c). Both $\alpha$ (ratio of the instantaneous reduced current to the instantaneous oxidized current) and $\beta$ (ratio of the initial reduced current to the initial oxidized current) represent the range of values observed in the experiments described in this work. 
For all steady state current ratios experimentally obtained in this work, the error is never greater than $5 \%$ for the total species concentration, which is reasonable over the course of this study. However, as seen from Figure 2 and Equation 8, as the diffusion coefficients between the neutral (reduced) and oxidized species begin to differ more, the error in estimated total species concentration increases.

\section{Electrode Stability}

Instability of the microelectrode can convolute analysis by masking the current signal resulting from homogeneous decay (e.g., solution-phase decomposition) with that from changes to the electrode surface. To determine if observed decay occurs because of electrode fouling or some other process on the electrode itself, we employ the ferrocene/ferrocenium redox couple as a model system, which is stable enough to be used as a reference potential in nonaqueous electrolytes. ${ }^{42}$ First, to avoid any possible complications from charging such as impurities generated at the counter electrode or temperature variations due to joule heating or mechanical heating from the stir plate, $5 \mathrm{mM}$ ferrocenium hexafluorophosphate was used directly. Initially, a carbon fiber microelectrode was used as the working electrode but, unexpectedly, the voltammetric response, both via steady state current and the shape of the voltammogram, suggested that a significant amount of decay occurred, which we attribute to physiochemical changes (e.g., fouling, degradation) on the electrode surface. Thus, we transitioned to a platinum microelectrode finding that, over the course of a 14-h experiment, the ferrocenium signal decayed by $c a .0 .5 \%$, which we believe is representative of the measurement resolution (Figure 3a). To ensure such decay did not occur in future tests, an additional measurement was taken with a freshly polished electrode at the 
end of the experiment and compared to the last scan. Unless specified otherwise, no difference was observed between the two voltammograms in all cases presented in this work.

\section{Temperature Variations}

The effects of solution cooling on microelectrode voltammograms mimic that of homogeneous decay, as increasing viscosity slows the diffusive transport of active species in solutions of lower temperature. This change results in a reduced steady state limiting current, which may be construed as a loss of active species concentration. It is therefore important to account for solution temperature during these measurements to avoid such artifacts. To investigate if electrochemical charging played a role in observed decay, an electrolyte solution containing $5 \mathrm{mM}$ ferrocene was oxidized in the bulk electrolysis cell, and the material fraction (the ratio of concentration at a given time to the initial concentration) was subsequently monitored with the platinum microelectrode (Figure 3b). Over the first $2.5 \mathrm{~h}$ of monitoring, the concentration dropped about $10 \%$ and then stabilized. We attribute this "decay" to solution cooling, which, in turn, impacts the molecular diffusivity via changes in temperature and viscosity as shown in the Stokes-Einstein relationship:

$$
D=\frac{k_{b} T}{6 \pi \mu a}
$$

Where $k_{b}$ is the Boltzmann constant $\left(1.38 \times 10^{-23} \mathrm{~J} / \mathrm{K}\right), T$ is the absolute temperature $(\mathrm{K}), \mu$ is the solution dynamic viscosity (Pa s), and $a$ is the hydrodynamic radius of the solute (m). The temperature and viscosity of the electrolyte solution, measured directly after electrochemical charging, were $30^{\circ} \mathrm{C}$ and $8.0 \mathrm{cP}$, respectively. The temperature increase is most likely due to a combination of mechanical stirring and joule heating from charging the solution. By the end of microelectrode measurements, the solution temperature dropped to $26^{\circ} \mathrm{C}$, in equilibrium with the glovebox temperature, and the viscosity rose to $8.7 \mathrm{cP}$. With this change in temperature and 
viscosity, the diffusion coefficient as determined by Equation 9, and consequently the steady state current, are expected to change by ca. $10 \%$, in agreement with the "decay" observed in the first $2.5 \mathrm{~h}$. As such, if the first time point used is $2.75 \mathrm{~h}$ after charging, $c a .1 \%$ decay occurs over the remaining $11 \mathrm{~h}$ of experiment (Figure 3c). Therefore, in all remaining analyses, the solution was left to cool for 2.5 hours before the initial measurement was recorded. 

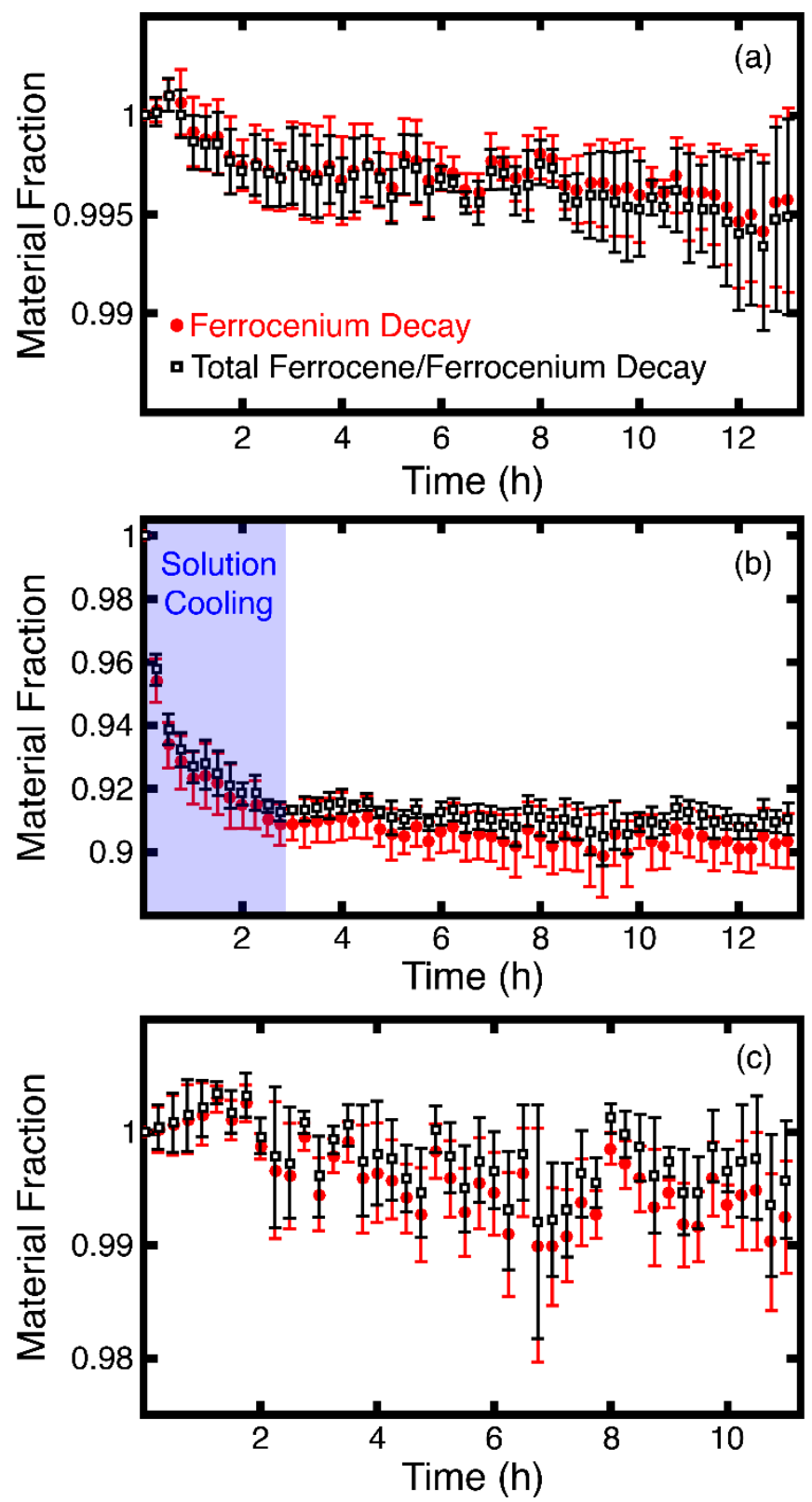

Figure 3: Relative concentration as a function of time for the ferrocene/ferrocenium redox couple for an initial solution of $5 \mathrm{mM}$ ferrocenium (a), an initial solution of $5 \mathrm{mM}$ ferrocene charged by bulk electrolysis (b), and the same data from (b) using the $2.75 \mathrm{~h}$ time point as the initial fraction of material in order to give the temperature of the solution time to equilibrate (c). For all plots the relative concentration of the ferrocenium (red circles) and the relative total concentration of redox active material (black squares) are shown, and the electrolyte used was 1 M LiTFSI in PC. All data was collected in triplicate. 


\section{$\mathrm{DBBB} / \mathrm{DBBB}{ }^{+}$Decay}

Confident in the insight this technique provides and its systemic sources of error explored using the model ferrocene/ferrocenium redox couple, we next study DBBB which, based on previous studies, is expected to undergo moderate decay. ${ }^{11,39}$ For the first test, $5 \mathrm{mM}$ of DBBB was used, the same concentration as the ferrocene experiments (Figure 4).

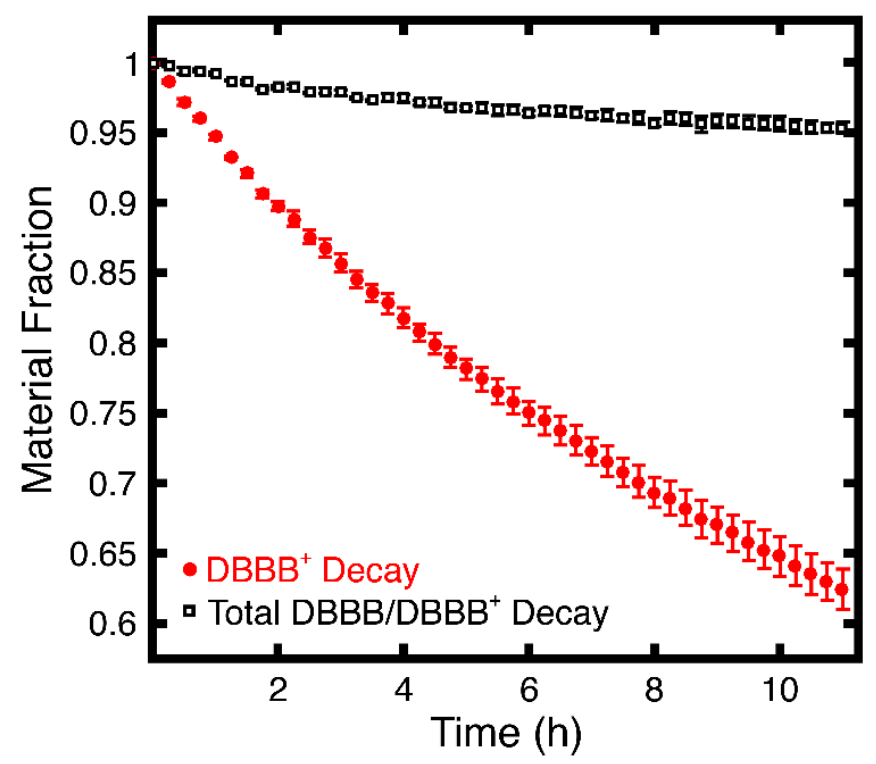

Figure 4: Relative concentration as a function of time for a $5 \mathrm{mM}$ solution of DBBB in $1 \mathrm{M}$ LiTFSI in PC. For all plots, the relative concentration of the radical cation (red circles) and the relative total concentration of redox active material (black squares) were collected in triplicate. Each solution was charged by bulk electrolysis.

At $5 \mathrm{mM}$, the charged species decays to about $60 \%$ of the initial value, but the total species only decays about $5 \%$, indicating that the majority of the charged DBBB decays to the neutral (reduced) species (DBBB). We note that, as detailed earlier, the percentage of total material decay is of a similar magnitude as the maximum measurement error for unequal diffusion coefficients. As such, we use SWV as a second electrochemical technique to confirm the total species decay. ${ }^{31,43}$ SWV is capable of determining the total concentration of an electroactive species in solution, but cannot easily resolve the respective individual concentrations of the reduced and oxidized species. 
Using the differential peak current value (the maximum current, proportional to the total concentration), the material decay was estimated and compared to the total species decay from the microelectrode experiments (Figure 5). The SWV measurements agree with the microelectrode measurements within the variation of the experiments, further validating this technique.

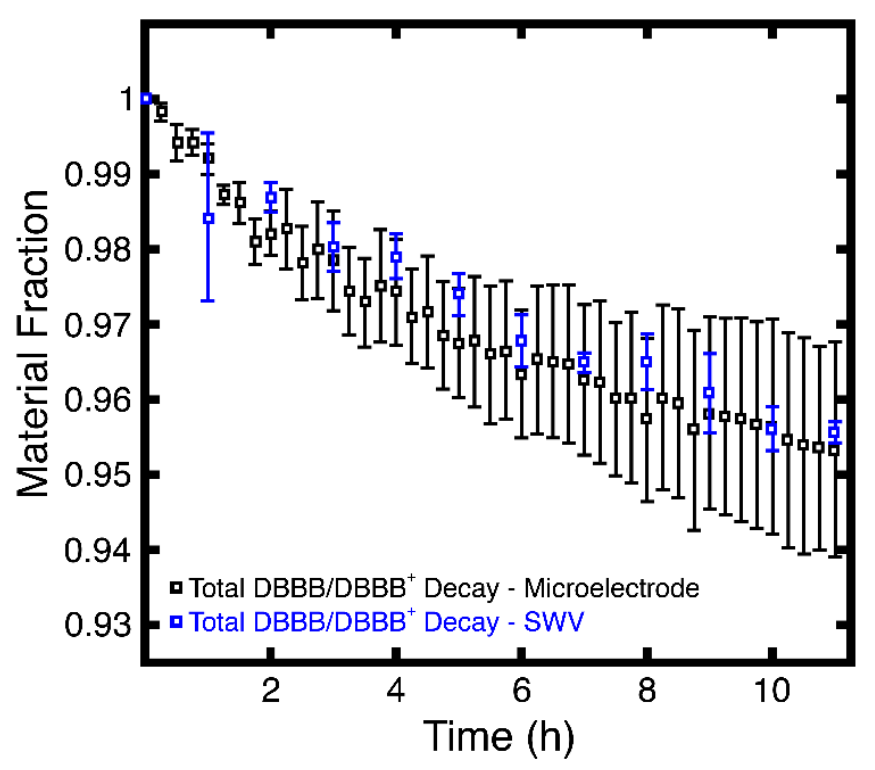

Figure 5: Relative total species concentration as a function of time for a $5 \mathrm{mM}$ solution of DBBB in $1 \mathrm{M}$ LiTFSI in PC collected by a microelectrode (black squares) every 15 min and collected by square wave voltammetry (blue squares) every hour. All measurements were collected in triplicate. Each solution was charged by bulk electrolysis.

As mentioned before, an advantage of microelectrode analysis is that the materials can be tested over a wide range of concentrations (from $1 \mathrm{mM}$ to at least $1 \mathrm{M}$ ), ${ }^{38}$ which may challenge other common analytical techniques. However, due to the method of charging, here electrochemical oxidation in a bulk electrolysis cell, the maximum concentration that could be tested was around $25 \mathrm{mM}$. For example, charging the $25 \mathrm{mM}$ solution took around $3 \mathrm{~h}$, while the charging time for many of the other solutions were about $1 \mathrm{~h}$. Thus, beyond $5 \mathrm{mM}$, DBBB was examined at 1, 3, and $25 \mathrm{mM}$. For this electrolyte composition, $1 \mathrm{mM}$ appears to be the lower concentration limit, as, below this value, the limiting current is on the same order of magnitude as 
the electrochemical noise of the experiment. Note that if the active species diffusion coefficient were larger, either based on the molecular structure, the choice of solvent, or the solution temperature, lower concentrations may be measurable. Figure 6 shows the compiled data of DBBB for all concentrations tested.

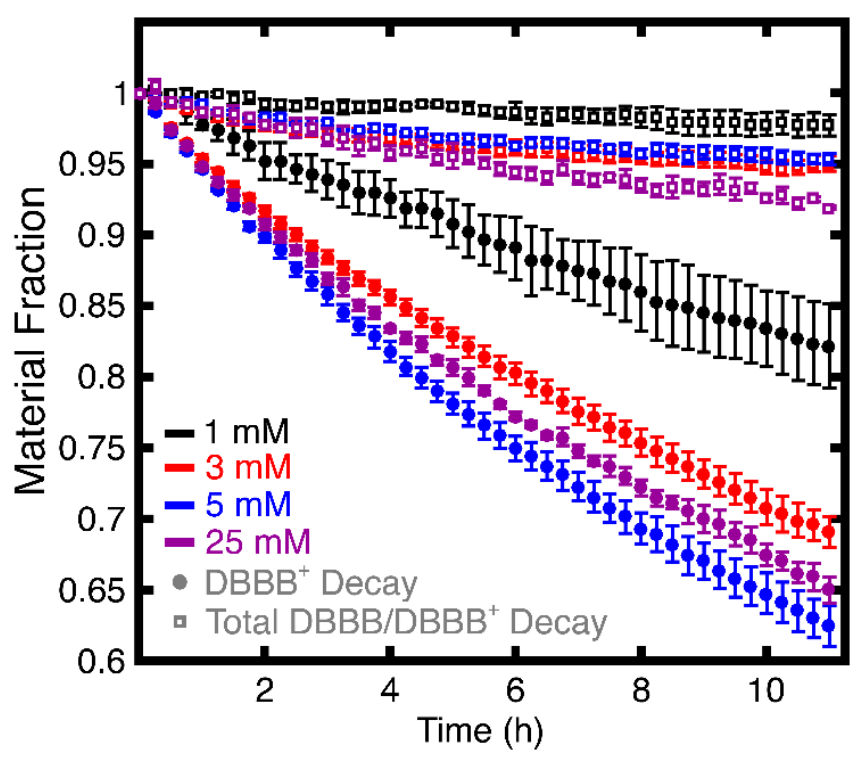

Figure 6: Relative concentration as a function of time for $1 \mathrm{mM}$ (black), $3 \mathrm{mM}$ (red), $5 \mathrm{mM}$ (blue), and $25 \mathrm{mM}$ (purple) solutions of DBBB in $1 \mathrm{M}$ LiTFSI in PC. The relative concentrations of the radical cation (circles) and the relative total concentrations of redox active material (squares) were collected in triplicate. Each solution was charged by bulk electrolysis.

In general, as the concentration increases, the decay rates of both the charged and total materials increase, indicating that the decay process is likely not first order. This increase is not observed when comparing the decay rate of the charged species at $5 \mathrm{mM}$ and $25 \mathrm{mM}$, further suggesting more complex decay kinetics. However, there may be some deviation due to the extra charging time at $25 \mathrm{mM}$ ( $3 \mathrm{~h}$ of charging rather than $1 \mathrm{~h}$ ), so additional decay occurs during this time that is unaccounted for. This appears to be a limitation of the charging method and electrochemical cell and could potentially be resolved through the application of more suitable 
electrochemical apparatus (e.g., flow cells) or starting with chemically-prepared oxidized species (chemical oxidization).

Because this technique yields concentration profiles based on the steady state currents, it is possible to obtain decay rates for the active species if the initial concentration is known. Figure 7 examines the decay rate of $\mathrm{DBBB}^{+}$alone for first and second order decay, both of which have precedent in the literature. ${ }^{11,22}$ Radical cations of dialkoxybenzenes decompose through multiple mechanisms, including dimerization, $O$-dealkylation via nucleophilic attack, and self-discharge through interactions with the solvent. ${ }^{11,22,39}$ To our knowledge, the decay of DBBB has not been extensively studied experimentally; however, based on studies with other, structurally similar dialkoxybenzenes, self-discharge and $O$-dealkylation are expected to be the primary modes of decomposition; dimerization is not expected to play a significant role in the decay of DBBB as the t-butyl groups sterically hinder this pathway. ${ }^{39}$ 


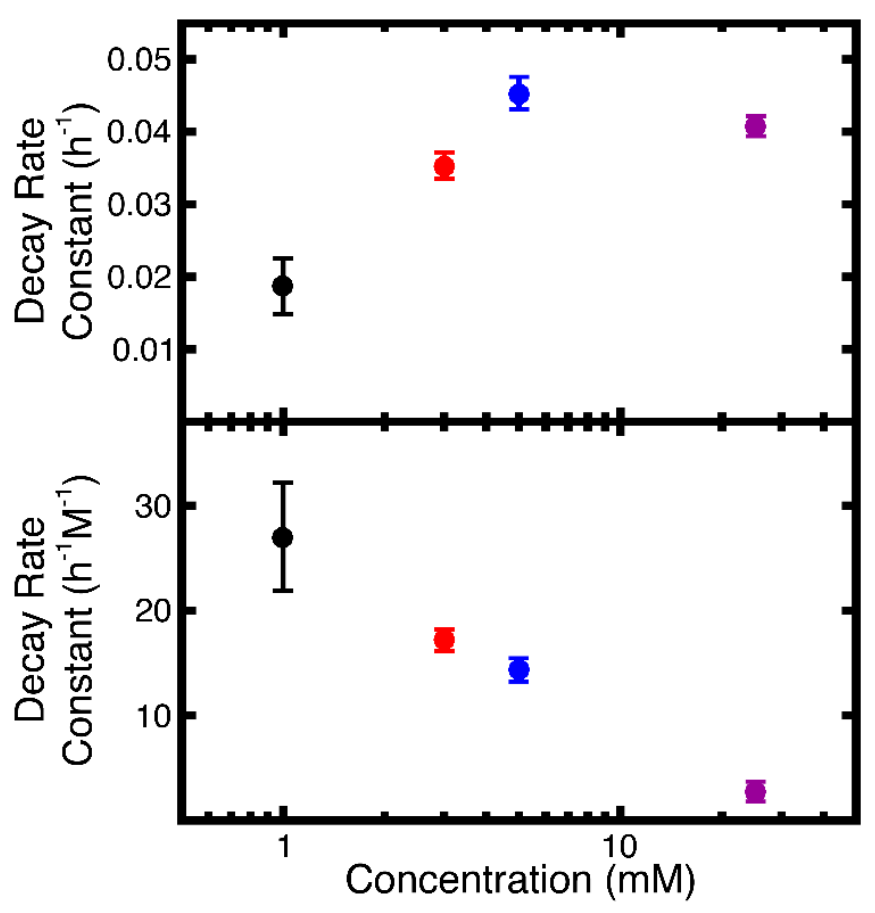

Figure 7: The decay rate as a function of concentration for $\mathrm{DBBB}^{+}$(charged species decay) in 1 M LiTFSI for the 4 concentrations tested (1, 3, 5, $25 \mathrm{mM}$ ), assuming a first order decay (top) and second order decay (bottom). All measurements were completed in triplicate.

The presence of multiple decay pathways implies that the true observed rate law is likely more complex than those directly explored in this work; indeed, the overall decomposition order of a different dialkoxybenzene changes as a function of conversion. ${ }^{22}$ Here, the difference in decay rate constants may indicate that different processes dominate at varying concentrations. However, the complex decay pattern observed in DBBB further highlights the value of this method; because the ratio of the concentration to the initial concentration of both charged and discharged species can be determined at any time, multiple modes of decay (specifically, self-discharge vs. $O$ dealkylation) may be deconvoluted. As such, this approach provides complementary insight into the different processes responsible for active species decay which, when combined with insight from spectroscopic techniques, can provide a more complete understanding of decay systems. 


\section{Conclusions}

Here we present an approach to simultaneously monitoring decay of charged and neutral species within a redox electrolyte using microelectrode voltammetry. To validate the proposed method, we first used the ferrocene/ferrocenium couple as a model system to identify and minimize sources of error (e.g., electrode surface and temperature changes). We then investigated DBBB, a moderately stable organic molecule, demonstrating an ability to detect decay of both the neutral and charged species across a range of concentrations corroborated with independent square wave voltammetry measurements.

While this is an initial demonstration focused on a relatively short-lived active species at a lower concentration and in 3-electrode cell, this methodology can be extended to higher concentration solutions, via improved charging methods (e.g., flow electrolysis), as well as to more stable compounds, via continuous or periodic monitoring over extended times. Increased temperature may also serve as a tool to enhance decay rates, enabling earlier detection, ${ }^{19}$ but caution should be taken to ensure new mechanistic pathways are not activated. Furthermore, this method can be applied to operating flow batteries where microelectrode probes could be used to monitor electrolyte state-of-charge and state-of-health in near real time. Finally, we note that, while this method alone cannot provide information on decomposition products, electroactive or otherwise, if used in conjugation with established analytical methods (e.g., UV-Vis, NMR, EPR),

electrochemical responses can be correlated to chemical composition, enabling richer analyses of complex electrochemical solutions of technological interest. 


\section{CRediT authorship contribution statement}

Jeffrey A. Kowalski: Conceptualization, Methodology, Investigation, Validation, Data curation, Visualization, Writing - original draft, Writing - review \& editing. Alexis M. Fenton Jr.: Visualization, Writing - original draft, Writing - review and editing. Bertrand J. Neyhouse: Visualization, Writing - original draft, Writing - review and editing. Fikile R. Brushett: Conceptualization, Project administration, Supervision, Writing - original draft, Writing - review and editing.

\section{Acknowledgements}

This work was supported as part of the Joint Center for Energy Storage Research, an Energy Innovation Hub funded by the U.S. Department of Energy, Office of Science, Basic Energy

Sciences. B.J.N gratefully acknowledges the National Science Foundation Graduate Research Fellowship Program under Grant No. 1122374. 


\section{List of variables and constants}

\begin{tabular}{|c|c|}
\hline & Hydrodynamic radius in solution (m) \\
\hline C & Concentration $\left(\mathrm{mol} / \mathrm{cm}^{3}\right)$ \\
\hline$c_{o}$ & Instantaneous concentration of the oxidized species $\left(\mathrm{mol} / \mathrm{cm}^{3}\right)$ \\
\hline$C_{o, i}$ & Initial concentration of the oxidized species $\left(\mathrm{mol} / \mathrm{cm}^{3}\right)$ \\
\hline$C_{r}$ & Instantaneous concentration of the reduced species $\left(\mathrm{mol} / \mathrm{cm}^{3}\right)$ \\
\hline$C_{r, i}$ & Initial concentration of the reduced species $\left(\mathrm{mol} / \mathrm{cm}^{3}\right)$ \\
\hline$D$ & Diffusion coefficient $\left(\mathrm{cm}^{2} / \mathrm{s}\right)$ \\
\hline$D_{o}$ & Diffusion coefficient of the oxidized species $\left(\mathrm{cm}^{2} / \mathrm{s}\right)$ \\
\hline$D_{r}$ & Diffusion coefficient of the reduced species $\left(\mathrm{cm}^{2} / \mathrm{s}\right)$ \\
\hline$F$ & Faraday’s constant $(96485 \mathrm{C} / \mathrm{mol})$ \\
\hline$F_{a c c}$ & Fraction of coulombs accessed in a single microelectrode scan \\
\hline$i_{s s}$ & Steady state current (A) \\
\hline$I_{s s, o}$ & Initial oxidizing steady state current (caused by the reduced species) (A) \\
\hline$i_{s s, o}$ & Instantaneous oxidizing steady state current (caused by the reduced species) (A) \\
\hline$I_{s, r}$ & Initial reducing steady state current (caused by the oxidized species) (A) \\
\hline$i_{s s, r}$ & Instantaneous reducing steady state current (caused by the oxidized species) (A) \\
\hline$k_{b}$ & Boltzmann's constant $\left(1.38 \times 10^{-23} \mathrm{~J} / \mathrm{K}\right)$ \\
\hline & Number of electrons transferred \\
\hline$Q_{c y c}$ & Maximum number of coulombs from a single microelectrode scan (C) \\
\hline Qsol & Number of coulombs in the solution from the microelectrode (C) \\
\hline & Radius of the microelectrode $(\mathrm{cm})$ \\
\hline$R_{c}$ & Ratio of the instantaneous total concentration to the initial total concentration \\
\hline$R_{c, a p p}$ & $\begin{array}{l}\text { Apparent ratio of the instantaneous total concentration to the initial total } \\
\text { concentration (assumes the diffusion coefficient of the reduced and oxidized species } \\
\text { are the same) }\end{array}$ \\
\hline$R_{c, \text { true }}$ & $\begin{array}{l}\text { True ratio of the instantaneous total concentration to the initial total concentration } \\
\text { (differs from } \mathrm{R}_{\mathrm{c} \text {,app }} \text { when the diffusion coefficient of the reduced species is different } \\
\text { from that of the oxidized species) }\end{array}$ \\
\hline$t_{c y c}$ & Time for one microelectrode scan (s) \\
\hline & Temperature (K) \\
\hline$V$ & Volume of solution in the microelectrode measurement $\left(\mathrm{cm}^{3}\right)$ \\
\hline$\alpha$ & Ratio of the instantaneous steady state currents of the reduced to oxidized species \\
\hline & Ratio of the initial steady state currents of the reduced to oxidized species \\
\hline & Ratio of the diffusion coefficients of the reduced to oxidized species \\
\hline & Fractional error of total concentration ratio from unequal diffusion coefficients \\
\hline & Solution dynamic viscosity (Pa s) \\
\hline
\end{tabular}




\section{References}

1. R. M. Darling, K. G. Gallagher, J. A. Kowalski, S. Ha, and F. R. Brushett, Energy Env. Sci, 7, 3459-3477 (2014).

2. J. A. Kowalski, L. Su, J. D. Milshtein, and F. R. Brushett, Curr. Opin. Chem. Eng., 13, 45-52 (2016).

3. C. G. Armstrong and K. E. Toghill, Electrochem. Commun., 91, 19-24 (2018).

4. J. Zhang, R. E. Corman, J. K. Schuh, R. H. Ewoldt, I. A. Shkrob, and L. Zhang, J. Phys. Chem. C, 122, 8159-8172 (2018).

5. L. Su, J. A. Kowalski, K. J. Carroll, and F. R. Brushett, in Rechargeable Batteries: Materials, Technologies and New Trends, Green Energy and Technology. Z. Zhang and S. S. Zhang, Editors, p. 673-712, Springer International Publishing (2015).

6. D. G. Kwabi, K. Lin, Y. Ji, E. F. Kerr, M.-A. Goulet, D. P. Tabor, D. A. Pollack, A. AspuruGuzik, R. G. Gordon, and M. J. Aziz, Joule, 2, 1894-1906 (2018).

7. Y. Ji, M.-A. Goulet, D. A. Pollack, D. G. Kwabi, S. Jin, D. De Porcellinis, E. F. Kerr, R. G. Gordon, and M. J. Aziz, Adv. Energy Mater., 9, 1900039 (2019).

8. V. Dieterich, J. D. Milshtein, J. L. Barton, T. J. Carney, R. M. Darling, and F. R. Brushett, Transl. Mater. Res., 5, 034001 (2018).

9. J. Huang, L. Su, J. A. Kowalski, J. L. Barton, M. Ferrandon, A. K. Burrell, F. R. Brushett, and L. Zhang, J. Mater. Chem. A, 3, 14971-14976 (2015).

10. C. S. Sevov, R. E. M. Brooner, E. Chénard, R. S. Assary, J. S. Moore, J. Rodríguez-López, and M. S. Sanford, J. Am. Chem. Soc., 137, 14465-14472 (2015).

11. J. Zhang, I. A. Shkrob, R. S. Assary, S. o. Tung, B. Silcox, L. A. Curtiss, L. Thompson, and L. Zhang, J. Phys. Chem. C, 121, 23347-23358 (2017).

12. A. Murali, A. Nirmalchandar, S. Krishnamoorthy, L. Hoober-Burkhardt, B. Yang, G. Soloveichik, G. K. Surya Prakash, and S. R. Narayanan, J. Electrochem. Soc., 165, A1193A1203 (2018).

13. M.-A. Goulet and M. J. Aziz, J. Electrochem. Soc., 165, A1466-A1477 (2018).

14. J. D. Milshtein, A. P. Kaur, M. D. Casselman, J. A. Kowalski, S. Modekrutti, P. L. Zhang, N. H. Attanayake, C. F. Elliott, S. R. Parkin, C. Risko, F. R. Brushett, and S. A. Odom, Energy Environ. Sci., 9, 3531-3543 (2016).

15. S. M. Laramie, J. D. Milshtein, T. M. Breault, F. R. Brushett, and L. T. Thompson, J. Power Sources, 327, 681-692 (2016). 
16. J. A. Kowalski, M. D. Casselman, A. P. Kaur, J. D. Milshtein, C. F. Elliott, S. Modekrutti, N. H. Attanayake, N. Zhang, S. R. Parkin, C. Risko, F. R. Brushett, and S. A. Odom, J. Mater.

Chem. A, 5, 24371-24379 (2017).

17. W. Duan, J. Huang, J. A. Kowalski, I. A. Shkrob, M. Vijayakumar, E. Walter, B. Pan, Z. Yang, J. D. Milshtein, B. Li, C. Liao, Z. Zhang, W. Wang, J. Liu, J. S. Moore, F. R. Brushett, L. Zhang, and X. Wei, ACS Energy Lett., 2, 1156-1161 (2017).

18. J. Huang, L. Cheng, R. S. Assary, P. Wang, Z. Xue, A. K. Burrell, L. A. Curtiss, and L. Zhang, Adv. Energy Mater., 5, 1401782 (2015).

19. C. S. Sevov, D. P. Hickey, M. E. Cook, S. G. Robinson, S. Barnett, S. D. Minteer, M. S. Sigman, and M. S. Sanford, J. Am. Chem. Soc., 139, 2924-2927 (2017).

20. C. S. Sevov, S. K. Samaroo, and M. S. Sanford, Adv. Energy Mater., 7, 1602027 (2017).

21. J. A. Kowalski, B. J. Neyhouse, and F. R. Brushett, Electrochem. Commun., 111, 106625 (2020).

22. J. Huang, B. Pan, W. Duan, X. Wei, R. S. Assary, L. Su, F. R. Brushett, L. Cheng, C. Liao, M. S. Ferrandon, W. Wang, Z. Zhang, A. K. Burrell, L. A. Curtiss, I. A. Shkrob, J. S. Moore, and L. Zhang, Sci. Rep., 6, 32102 (2016).

23. J. Zhang, Z. Yang, I. A. Shkrob, R. S. Assary, S. on Tung, B. Silcox, W. Duan, J. Zhang, C. C. Su, B. Hu, B. Pan, C. Liao, Z. Zhang, W. Wang, L. A. Curtiss, L. T. Thompson, X. Wei, and L. Zhang, Adv. Energy Mater., 7, 1701272 (2017).

24. J. Zhang, J. Huang, L. A. Robertson, R. S. Assary, I. A. Shkrob, and L. Zhang, J. Phys. Chem. C, 122, 8116-8127 (2018).

25. E. W. Zhao, T. Liu, E. Jónsson, J. Lee, I. Temprano, R. B. Jethwa, A. Wang, H. Smith, J. Carretero-González, Q. Song, and C. P. Grey, Nature, 579, 224-228 (2020).

26. A. P. Kaur, N. E. Holubowitch, S. Ergun, C. F. Elliott, and S. A. Odom, Energy Technol., 3, 476-480 (2015).

27. M. I. Montenegro, M. A. Queirós, and J. L. Daschbach, Eds., Microelectrodes: Theory and Applications, Kluwer Academic Publishers, (1991).

28. R. G. Compton and C. E. Banks, Understanding Voltammetry, 2nd ed., World Scientific, (2014).

29. A. M. Bond, The Analyst, 119, 1-21 (1994).

30. L. Su, M. Ferrandon, J. A. Kowalski, J. T. Vaughey, and F. R. Brushett, J. Electrochem. Soc., 161, A1905-A1914 (2014). 
31. A. J. Bard and L. R. Faulkner, Electrochemical Methods: Fundamentals and Applications, 2nd ed., John Wiley \& Sons. Inc., New York, (2001).

32. S. M. Radke and E. C. Alocilja, Biosens. Bioelectron., 20, 1662-1667 (2005).

33. E. Llaudet, N. P. Botting, J. A. Crayston, and N. Dale, Biosens. Bioelectron., 18, 43-52 (2003).

34. D. Pletcher and E. M. Valdes, Anal. Chim. Acta, 246, 267-273 (1991).

35. D. Pletcher and S. Sotiropoulos, Anal. Chim. Acta, 322, 83-90 (1996).

36. N.-H. Yeh, M. Medcalf, and K. D. Moeller, J. Am. Chem. Soc., 140, 7395-7398 (2018).

37. M. D. Graaf, B. V. Marquez, N.-H. Yeh, S. E. Lapi, and K. D. Moeller, ACS Chem. Biol., 11, 2829-2837 (2016).

38. C. Stolze, J. P. Meurer, M. D. Hager, and U. S. Schubert, Chem. Mater., 31, 5363-5369 (2019).

39. L. Zhang, Z. Zhang, P. C. Redfern, L. A. Curtiss, and K. Amine, Energy Environ. Sci., 5, 8204-8207 (2012).

40. A. Lewandowski, M. Osińska, A. Swiderska-Mocek, and M. Galinski, Electroanalysis, 20, 1903-1908 (2008).

41. J. Newman and K. E. Thomas-Alyea, Electrochemical Systems, 3rd ed., John Wiley \& Sons. Inc., (2004).

42. R. R. Gagne, C. A. Koval, and G. C. Lisensky, Inorg. Chem., 19, 2854-2855 (1980).

43. Y. Oh, M. L. Heien, C. Park, Y. M. Kang, J. Kim, S. L. Boschen, H. Shin, H. U. Cho, C. D. Blaha, K. E. Bennet, H. K. Lee, S. J. Jung, I. Y. Kim, K. H. Lee, and D. P. Jang, Biosens.

Bioelectron., 121, 174-182 (2018). 